\title{
Prediction of ohmic losses in miter bend polarizers
}

\author{
D. Wagner ${ }^{1}$, F. Leuterer ${ }^{1}$, W. Kasparek ${ }^{2}$, C. Lechte ${ }^{2}$, J. Stober ${ }^{1}$ and ASDEX Upgrade \\ ${ }^{1}$ Max-Planck-Institut für Plasmaphysik, Boltzmannstr.2, D-85748 Garching, Germany \\ ${ }^{2}$ Institut für Grenzflächenverfahrenstechnik und Plasmatechnologie, Universität Stuttgart, Pfaffenwaldring 31, D-70569 \\ Stuttgart, Germany
}

The polarization required to couple the output beam of Electron Cyclotron Resonance Heating (ECRH) systems efficiently to the plasma is usually provided by a set of two grooved polarizer mirrors $[1,2]$. These polarizers can convert the linear polarization of a gyrotron output beam into any arbitrary output polarization. Since the ohmic losses of these mirrors can significantly exceed the losses of plane mirrors (up to 3-4 times, depending on their groove geometry and depth), they are considered as one of the most critical elements in high power long-pulse ECRH transmission lines with respect to cooling. This is especially the case when such mirrors are incorporated into miter bends in closed evacuated corrugated waveguide transmission lines like the ones for ITER. This is due to the limited diameter of the mirror and the centrally peaked power profile of the $\mathrm{HE}_{11}$ mode.

The ohmic loss of polarizer mirrors has been investigated experimentally at high power experiments using test mirrors mounted in a miter bend of an existing high-power transmission line (Fig.1). For the high-power tests several polarizer mirrors were fabricated out of stainless steel (VA 1.4311) and copper. First tests were performed using stainless steel mirrors in order to get an easily measurable high temperature difference together with a slow heat transfer towards the mirror edges. The thickness of the test mirrors was $5 \mathrm{~mm}$. The polarizer mirrors have a sinusoidal corrugation with depth $0.8 \mathrm{~mm}$ and $0.57 \mathrm{~mm}$ respectively, and a corrugation period of $1.07 \mathrm{~mm}$. For comparison a plane disk, also with a thickness of $5 \mathrm{~mm}$ of the same material, was prepared. The mirror diameter is $140 \mathrm{~mm}$ covering the $87 \mathrm{~mm}$ inner diameter of the corrugated waveguides. All mirrors under test were equipped with a PT-100 platinum resistance temperature detector at the center of the back side whose dimension is $2 \times 2 \mathrm{~mm}$, thus averaging over 2 corrugation periods. The sensitivity is $6^{\circ} \mathrm{K} / \mathrm{V}$. At the end of each AUG transmission line the beam is directed into a short-pulse calorimetric load via another miter bend with directional coupler. For the polarizer tests one of the gyrotrons was operated at $140 \mathrm{GHz}$ into such a calorimetric load. For the tests we applied an output power of $375 \mathrm{~kW}$ with a pulse length of $50 \mathrm{~ms}$ resulting in easily measureable temperature differences of several degrees. The response time of the PT-100 detectors on the back side is about 0.5 s. Fig. 2 shows a typical temperature signal following a $50 \mathrm{~ms}$ gyrotron pulse. The maximum is reached $4 \mathrm{~s}$ after the pulse start. For the central position of the sensor there is only a very small radial temperature gradient, and therefore the radial heat transfer is small compared to the heat transfer to the backside, as can be seen in the slow decay of the temperature signal after reaching the maximum compared to the fast rise of the temperature right after the $50 \mathrm{~ms}$ gyrotron pulse. The maximum temperature can thus be taken as a measure of the energy deposited on the front mirror surface by the beam. After each pulse the mirror was cooled back to room temperature by pressurized air. Using the polarizer mirrors in the matching optics unit any required input polarization onto the miter bend mirror under test can be set

A strategy to minimize the losses for given mirror geometries has been developed [3]. For the usually applied polarizer systems consisting of a polarization rotator (groove depth : $\sim \lambda_{0} / 4$, with $\lambda_{0}$ being the free space wavelength) together with an elliptical polarizer (groove depth $: \sim \lambda_{0} / 8$ ) the combination of polarizer angles exhibiting the lowest losses can be selected of four possible solutions. Fig.3 shows a comparison between measured and calculated losses of the four solutions for a polarization rotator. For other polarizer systems the number of solutions can be less [4]. The losses in Fig. 4 are given by a normalized absorption

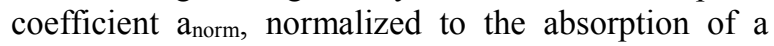
plane mirror with perpendicular incidence. Additionally the signal from a directional coupler at the end of the transmission line is plotted which remains approximately constant, thus verifying the same output polarization for all four solutions.

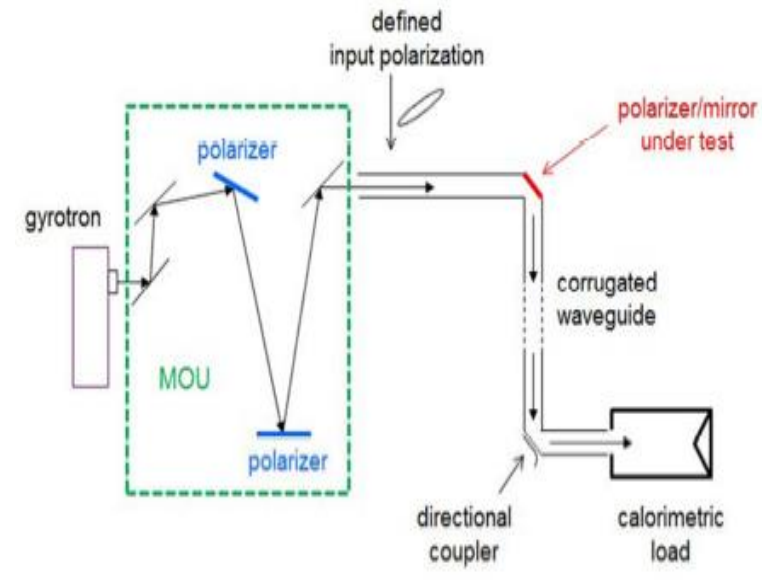

Fig. 1. Measurement setup. 


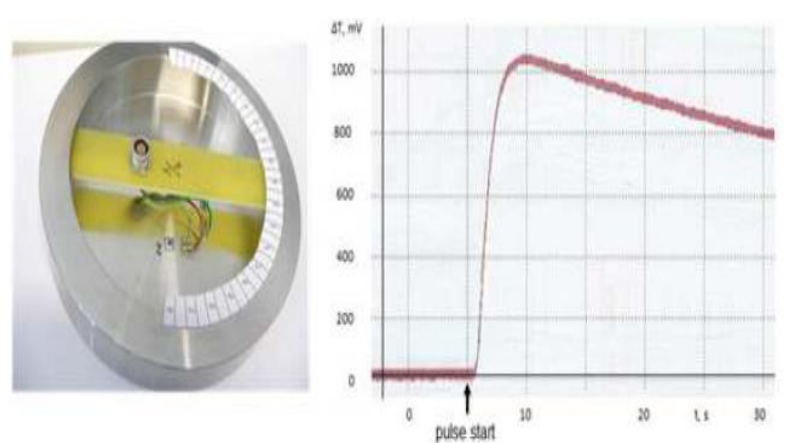

Fig. 2. Test mirror with PT-100 detectors (left) and measured temperature sequence of the central PT-100 detector (right).

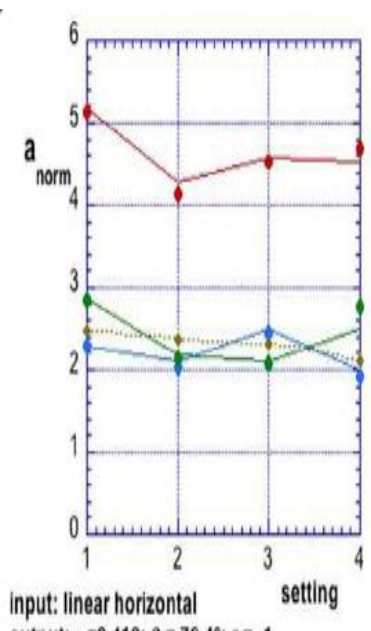

output: $\varepsilon=0.418 ; \beta=76.4^{\circ} ; r=.1$

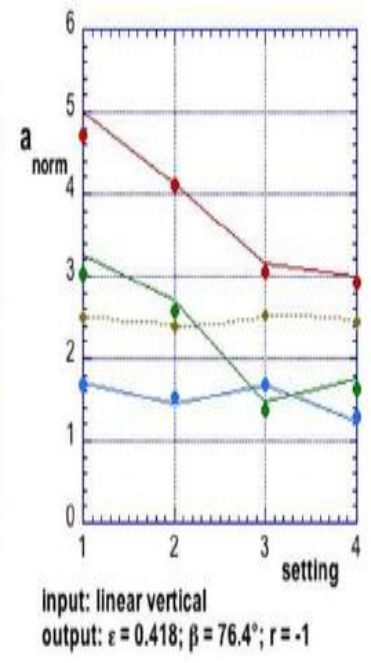

output: $\varepsilon=0.418 ; \beta=76.4^{\circ} ; r=-1$

Fig.3 Measured and calculated normalized losses for the four possible settings leading to an elliptical output polarization. Left: horizontal linear input; right: vertical linear input. Dots: experimental data; lines: model calculation

\section{References}

1. J.L. Doane et al., Polarizer miter bends for highpower microwave transmission: Ohmic loss and cooling, Fusion Engineering and Design, Vol. 102 (2016), 99-107.

2. D. Wagner, F. Leuterer, Broadband polarizers for high power multi-frequency ECRH systems, Journal of Infrared, Millimeter and Terahertz Waves, Vol. 26 (2005), 163-172.

3. D. Wagner et al., Minimization of the ohmic loss of grooved polarizer mirrors in high-power ECRH systems, Journal of Infrared, Millimeter and Terahertz Waves, Vol. 37 (2016), 45-54.

4. F. Leuterer et al., Experimental study of Ohmic losses of polarizer mirror system, Journal: EPJ Web of Conferences, 2017, Volume 149, Page 03002, DOI: 10.1051/epjconf/201714903002. 